\title{
Asociatividad y liderazgo con productores agropecuarios del Departamento del Quindío
}

\section{Associativity and leadership with agricultural producers of the Department of Quindío}

\author{
DOI: $10.46932 / \mathrm{sfjdv2n4-034}$
}

Received in: March 1st, 2021

Accepted in: May 30th, 2021

\author{
Leidy Carolina Cardona Hernández \\ Magister en Desarrollo Regional y Planeación del Territorio \\ Programa Trabajo Social, Facultad Ciencias Humanas y Bellas Artes. \\ Universidad del Quindío. Carrera 14 calle 12 N. Armenia. Colombia \\ E-mail: lccardona@uniquindio.edu.co
}

\author{
Henry Reyes Pineda \\ PhD Ingeniería Química \\ Programa Tecnología en procesos Agroindustriales. Facultad Ciencias Agroindustriales \\ Universidad del Quindío. Carrera 14 calle 12 N. Armenia \\ E-mail: hreyes@uniquindio.edu.co \\ Ramón Gutiérrez Robledo \\ Ingeniero Agrónomo \\ Programa Tecnología Agropecuaria, Facultad Ciencias Agroindustriales \\ Universidad del Quindío. Carrera 14 calle 12 N. Armenia \\ E-mail: rgutierrez@uniquindio.edu.co
}

\section{RESUMEN}

El fortalecimiento organizacional de las asociaciones de productores del Departamento del Quindío en cabeza de sectores como el porcícola, cunícula, hortofrutícola, son ejes estratégicos para la Universidad del Quindío y con el que se busca que los productores agropecuarios de los municipios, tengan la capacidad de aplicar los procesos tecnológicos en la transformación vegetal, cárnica y láctea, a su vez creando y manteniendo una adecuada marca de sus productos y empresas, situación que beneficiara el sostenimiento de estos, junto con su plan de negocio, en el mercado comercial. La base para fundamentar dichos procesos es: liderazgo, trabajo en equipo, asociatividad, en aras del desarrollo local y regional. Esto como resultante del trabajo interdisciplinar que la Uniquindío ha realizado desde sus Vicerrectorías, Facultades y programas como lo son Vicerrectoría de Investigaciones, Vicerrectoría de Extensión y Desarrollo Social, las Facultades de Ciencias Agroindustriales, Ciencias Económicas y administrativas y la Ciencias Humanas y Bellas Artes y los Programas de Tecnología Agropecuaria, Tecnología Agroindustrial, Ingeniería de Alimentos, Economía, Trabajo social y Comunicación social-Periodismo, que a través de sus interacción han logrado las aplicaciones tecnológicas en la trasformación de materias primas que se generan como resultado de un proceso de indagación e investigación en los procesos de formulación de empresa de los productores agropecuarios de los municipios de del Departamento, particularmente de los municipios de Filandia, Circasia, Quimbaya, Pijao, Buenavista, Génova y Córdoba.

Palabras Clave: Asociatividad, trasformación, valor agregado, empresarización, desarrollo social. 


\section{ABSTRACT}

The organizational strengthening of the associations of producers of the Department of Quindío at the head of sectors such as pig, cunícula, hortofrutícola, are strategic axes for the University of Quindío and with which it is sought that the agricultural producers of the municipalities, have the capacity to apply the technological processes in the vegetable, meat and dairy processing, in turn creating and maintaining an adequate brand of their products and companies, a situation that will benefit the sustainability of these, together with their business plan, in the commercial market. The basis to base these processes is: leadership, teamwork, associativity, in the interest of local and regional development. This as a result of the interdisciplinary work that Uniquindío has carried out from its Vice-Rectories, Faculties and programs such as Vice-Rector for Research, Vice-Rector for Extension and Social Development, the Faculties of Agroindustrial Sciences, Economic and Administrative Sciences and the Human Sciences and Fine Arts and the Programs of Agricultural Technology, Agroindustrial Technology, Food Engineering, Economy, Social Work and Social Communication-Journalism, which through their interaction have achieved technological applications in the transformation of raw materials that are generated as a result of a process of inquiry and research in business formulation processes of agricultural producers in the municipalities of the Department, particularly in the municipalities of Filandia, Circasia, Quimbaya, Pijao, Buenavista, Genoa and Córdoba..

Keywords: Associativity, transformation, added value, entrepreneurship, social development.

\section{INTRODUCCIÓN}

La preocupación de las organizaciones y comunidades por el bienestar y buen vivir de sus integrantes ha aumentado, se ha observado como esto repercute directamente en el desempeño de sus trabajos, ello ha generado que los conceptos de trabajo en red, trabajo en equipo, liderazgo, cooperativismo, asociatividad, desarrollo local y competitividad, aparezcan en este escenario y emerjan como un elemento vital dentro de las organizaciones; a su vez, conlleva a generar empoderamiento y fortalecer los capitales intangibles en los diversos contextos sociales (Martelli, 2020).

En el marco de las acciones que ha emprendido la Vicerrectoría de Investigaciones de la Universidad del Quindío, la cual busca fomentar alianzas estratégicas con el sector productivo del Departamento, respondiendo a las necesidades locales y regionales, se han diseñado capacitaciones y talleres para diversas asociaciones y cooperativas, desde un trabajo articulado e interdisciplinario que adelantan la facultad de Ciencias Agroindustriales, la Facultad de Ciencias Humanas y Bellas Artes y la Facultad de Ciencias Administrativas, Económicas y Contables en el marco del proyecto "fortalecimiento organizacional de las asociaciones y cooperativas productoras de los municipios de Filandia, Circasia, Quimbaya, Pijao, Buenavista, Génova y Córdoba"

Desde dicha perspectiva, se propuso como objetivo apoyar a los productores de dichos municipios para que puedan asociarse y organizarse en torno a sus vocaciones productivas, a través del reconocimiento de su entorno, de transferencia de conocimientos y tecnológica en sectores cárnicos y lácteos, mediante temas estratégicos que hacen parte de la asociatividad, a su vez integrar estrategias 
lúdico-metodológicas que le permitan avanzar hacia un trabajo colaborativo, de bienestar de las comunidades, de desarrollo local a través de la construcción de marca y de espacios de relaciones públicas.

Se tuvo como reto, abordar los conceptos y aspectos relevantes que contribuyen a fortalecer las asociaciones, es una herramienta significativa en la medida que permite abordar categorías conceptuales que son indispensables para avanzar hacia el trabajo mancomunado al interior de las cooperativas y organizaciones en los diferentes municipios, a su vez, permite dar una lectura al contexto y brindar elementos necesarios que exige la globalidad, reflexionar frente a estudios de caso acerca de las dificultades que actualmente experimentan las Asociaciones productoras en el Quindío cimentado en: Articulación en red, liderazgo asociativo y transformacional, trabajo en equipo y organizaciones saludables que aprenden.

El resultado de este proceso, surge gracias al acercamiento con los sujetos participes del proyecto, un gremio de asociados que buscan un mejoramiento continuo que se traduzca en el bien común y de cohesión social, durante el proceso, el apoyo de metodologías interactivas y técnicas grupales permitieron obtener mejores resultados en el cambio de actitudes y comportamientos de los integrantes de las asociaciones y cooperativas productoras.

\section{JUSTIFICACIÓN}

Es necesario entender el territorio como un sistema abierto y complejo, para tener la posibilidad de empezar a hablar de desarrollo, no como una simple suma aleatoria de formas geográficas y de resultados parciales, más bien como un proceso descentralizado que se teje desde la persona humana, donde tiene en realidad sus orígenes, es ella la encargada de proporcionar una verdadera identidad al territorio, de "construir una sociedad que genere capacidad de lenguaje, para establecer relaciones abstractas y sobre todo simbólicas". (Boissier, 2010). Es el inicio de una construcción social la que permitirá la potenciación del ser humano para transformarse y poder llegar a ese estado de asociatividad con su entorno, es el primer paso, además fundamental para comprender el sentido del desarrollo.

Se tiene la necesidad de colocar al ser humano como eje central en la transformación del territorio, de recuperar aquella identidad que se ha ido desvaneciendo, de apropiarse del contexto y crear iniciativas que conlleven a potenciar lo local, en este sentido no solo cobra importancia mirar la aplicabilidad de los diferentes sistemas económicos en determinado lugar, es necesario pensar como desde dichas realidades es posible impulsar los sistemas económicos endógenos. 
Es de vital importancia para la construcción del territorio la actuación sinergética de los actores y por supuesto la participación, que se convierte en un instrumento de empoderamiento y equidad social que debe involucrar a todos los actores.

A partir de lo expuesto, se trazan dos caminos determinantes para abordar el proyecto, un corpus teórico que fundamenta su accionar y uno metodológico que permite el acercamiento a la población y el cumplimiento de los indicadores trazados.

Desde la contextualización teórica-conceptual se trabajan categorías como la asociatividad, concebida como un atributo comunitario que engloba aspectos de la vida social como son redes sociales, normas y confianza mutua, las cuales son formas más efectivas de alcanzar objetivos y metas comunes de los individuos que gozan de ese capital social” (Villarán, 2000).

La asociatividad es un mecanismo mediante el cual personas o empresas, manteniendo su independencia jurídica y operativa, deciden voluntariamente llevar a cabo procedimientos en conjunto para llegar a un fin común, pero en realidad va más allá de una figura jurídica, esta herramienta es entonces la alternativa para que el pequeño y mediano productor del sector agropecuario, incremente sus ingresos y mejore su calidad de vida; mediante la asociatividad se estandarizan procesos de producción, se minimizan los intermediarios, los cuales en la mayoría de casos son los que absorben las ganancias, haciendo que el productor y el consumidor final, se vean afectados económicamente, así mismo. (Departamento Nacional de Planeación DNP, 2007)

Por su parte, el liderazgo entendido desde Sánchez, \&Vázquez (2010) como;

"la capacidad de comunicarse con un grupo de personas, influir en sus emociones para que se dejen dirigir, compartan las ideas del grupo, permanezcan en el mismo, y ejecuten las acciones o actividades necesarias para el cumplimiento de uno o varios objetivos. Aquella influencia que es positiva para todo el grupo, es decir, que ayuda al grupo a llevar a término su propósito, a conseguir objetivos, a mantener un buen funcionamiento, y a adaptarse a su entorno.

Como elemento fundamental del proyecto, se aborda el liderazgo asociativo el cual emerge como una forma específica para alcanzar niveles adecuados de gestión, desarrollo organizacional y competitividad al interior de las asociaciones, con la descentralización se adquiere una nueva forma de concebir las acciones y se empiezan a redefinir los procesos donde se vinculan nuevos actores, el sector productivo y las comunidades locales se convierten en agentes sumamente importantes en este escenario, está orientada a vincularse de manera recíproca con la comunidad, trazando objetivos colectivos y logros duraderos, las características que se abordan en esta categoría conceptual, se exponen en la Figura 1. 
Figura 1. Elementos Constitutivos del Liderazgo Asociativo.

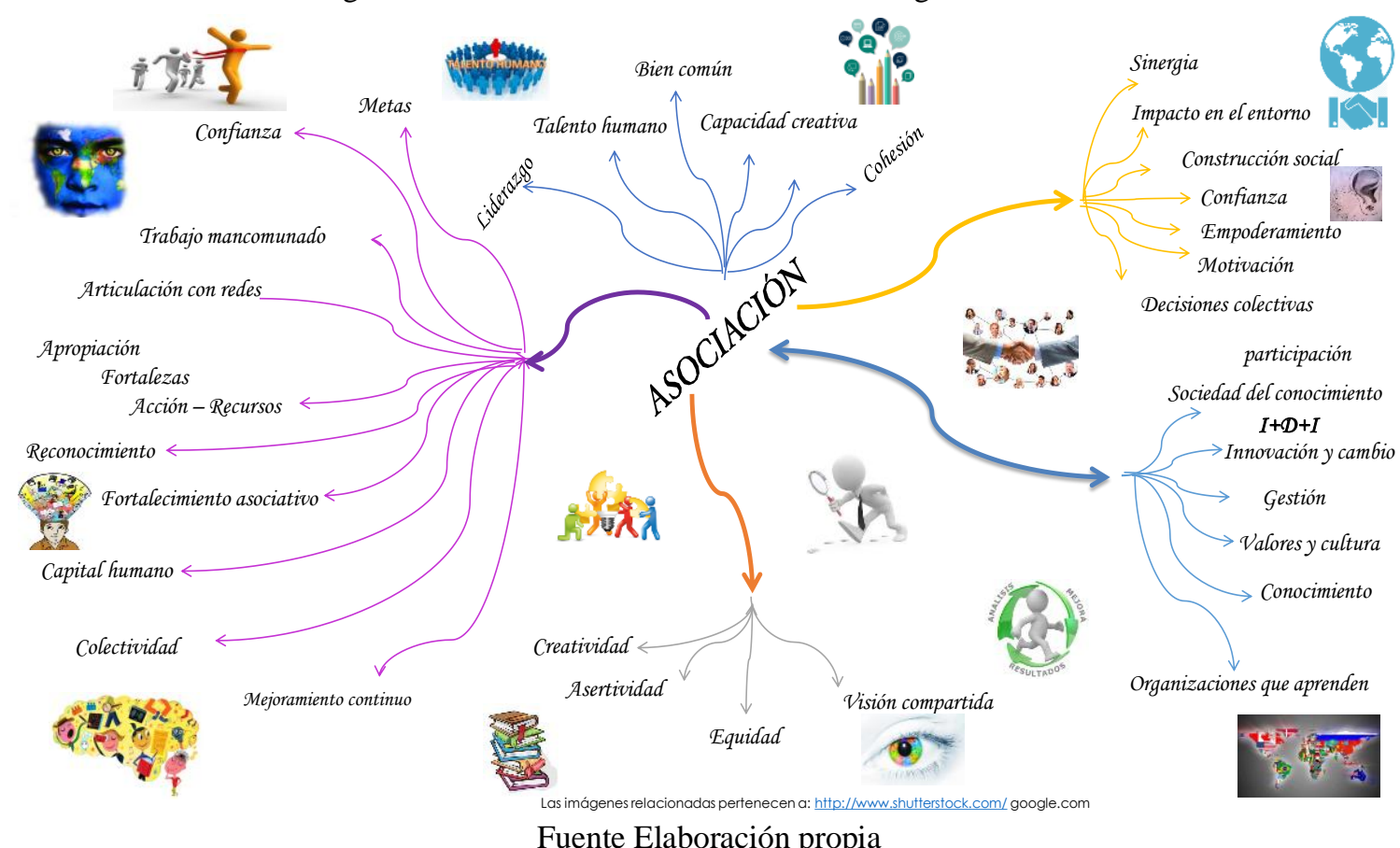

De otro lado, la competitividad es la capacidad de tomar decisiones económicas de calidad que generen valor y produzcan buenos resultados en la economía globalizada (desarrollo propio, no estándar e incluyente).

Es un escenario regional especifico, la competitividad es la capacidad que tienen sus habitantes (individual y colectivamente, desde los distintos ámbitos de su actividad en la sociedad) de identificar, crear y aprovechar, con el apoyo del estado, en beneficio de todos y en forma permanente y sostenible, ventajas económicas de un mercado global, creando valor agregado, a partir del aprovechamiento y mejoramiento de sus activos tangibles (recursos naturales y capital ambiental) e intangibles (capital humano y social, cultura e instituciones, sociedad del conocimiento)”. (Rodríguez, 2012. p.99).

\section{METODOLOGÍA}

El proyecto planteó un alcance exploratorio- descriptivo ya que se trazó como objetivo fortalecer la asociatividad y competitividad en los municipios de Filandia, Circasia, Quimbaya, Pijao, Buenavista, Génova y Córdoba, reconocidos por su trayectoria agrícola en el Departamento del Quindío, para cumplir dicho objetivo fue indispensable conocer las realidades de este sector productivo desde los sujetos, identificar las fortalezas y opciones de mejora para de esta manera propiciar escenarios de participación colectiva que mejoren la producción del sector, le permita generar redes y utilizar herramientas tecnológicas sostenibles. Las técnicas se expresan en la Figura 2. 
Figura 2. Técnicas cualitativas.

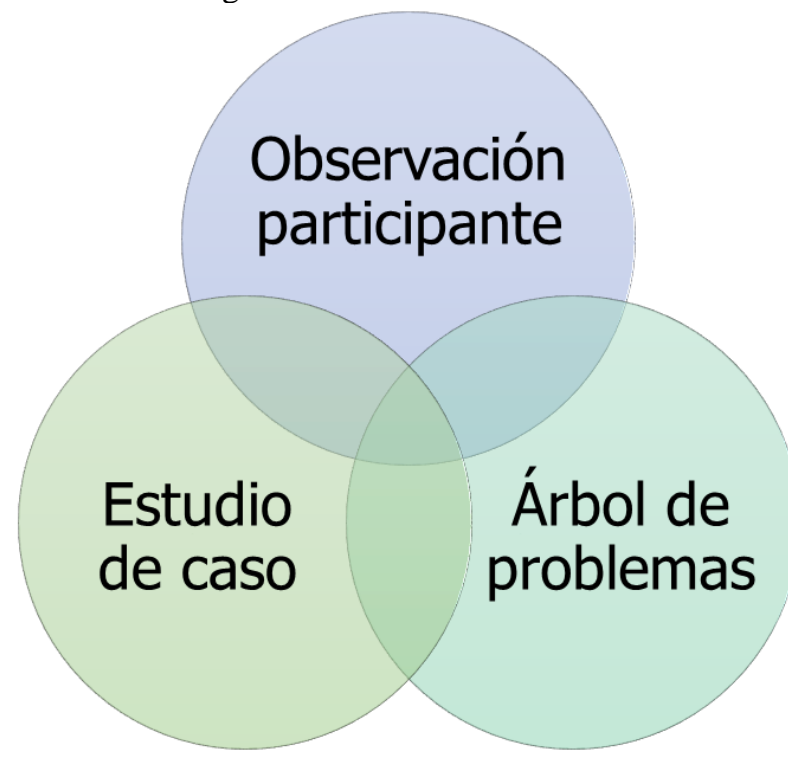

Fuente Elaboración propia

\subsection{ACTIVIDADES}

a. Presentación de la propuesta.

- Dinámica de presentación: Tendedero social

b. Trabajo en red

- $\quad$ Conceptos básicos

- $\quad$ Sentido de pertenencia

- Relaciones interpersonales

c. Trabajo colaborativo/trabajo en equipo

- $\quad$ Mapa de sueños

- Diccionario alternativo para una mayor participación

d. Liderazgo

- Comunicación Asertiva

- ¿Aquí quién manda? Y ¿Aquí como se manda?

- $\quad$ Toma de decisiones y resolución de problemas.

e. Cooperativismo Vs. Asociatividad

f. Desarrollo local

- Concepto de territorio

g. Check-out

- Qué aprendimos y para dónde vamos

- $\quad$ Encuesta de satisfacción 


\section{RESULTADOS}

Reconocer estas realidades locales de manera conjunta desde las categorías de análisis trabajadas a través de los talleres orientados en la Universidad el Quindío y el trabajo de campo, permitieron en buena medida crear compromisos consensuados en las Asociaciones, resaltando la importancia de avanzar hacia un trabajo mancomunado entre los diferentes actores considerando estos elementos como garantes a la hora de construir territorio.

La base que sostiene los proyectos debe estar mediado por el fortalecimiento de las capacidades para trabajar en equipo, reconocerse a sí mismo como Asociación permite transformar, crecer y hacer crecer; el impacto durante la ejecución del proyecto ha sido importante puesto que es una temática que no se había trabajado antes y que es realmente necesaria, allí se identifican actores líderes que tienen objetivos comunes que permiten fortalecer los lazos asociativos, se reconoce la otredad, se dan las bases necesarias para presentar proyectos y propuestas que mejoren el bienestar social y que por tanto incidan de manera positiva en el territorio.

La capacidad local de un territorio esta mediada por el fortalecimiento del capital humano y social, desde esta perspectiva es indispensable robustecer los procesos de participación comunitaria entendida como una toma de conciencia colectiva, de promoción de formas asociativas y organizativas que facilitan el bien común.

Con el proyecto se hicieron aportes importantes frente a la importancia que representa trabajar sobre objetivos comunes, que den respuesta a las expectativas de la comunidad y sean capaces de provocar impactos positivos en la región. Las fortalezas a nivel local permiten sin duda un posicionamiento a nivel global, por ello el reconocimiento de las capacidades endógenas conllevan a consolidar proyectos colectivos que permiten dar respuesta a sus necesidades y aprovechar las oportunidades dadas desde diversos campos.

El tener como referente que esta apuesta hacia lo común es un ejercicio de conciencia que debe hacerse desde cada uno de los actores participes en las diferentes asociaciones en cabeza de sus directivas y de sus integrantes, permite consolidar plataformas de liderazgo y brinda oportunidades para conformar clúster asociativo al interior de las asociaciones una apuesta fundamental para la avanzar hacia del Desarrollo Regional y Territorial.

Frente a lo expuesto se dio un avance importante, se identificaron algunos de los conflictos dados internamente que obstaculizan de alguna manera su desarrollo óptimo, se identificaron los líderes y se reconoció la importancia de trabajar desde la construcción de Comités, entre ellos se destacan los siguientes: Comité de salud, deporte, gestión de proyectos. 


\subsection{RESUMEN DE LAS ACTIVIDADES EJECUTADAS CON EL SECTOR PRODUCTIVO EN LOS} MUNICIPIOS.

Se realizó capacitación a jóvenes rurales, hijos de diversas asociaciones productoras de los municipios de municipios de Filandia, Quimbaya, Circasia, Buenavista, Pijao, Córdoba y Génova; estos se desarrollaron en varias sesiones: primero en identidad, asociatividad y liderazgo, y el segundo de proyección de la identidad y contexto cultural por medio de las herramientas digitales, que conllevan al empoderamiento de sus marcas propias, ya que en su gran mayoría son parte integral del desarrollo productivo de su entorno local y regional.

Dentro de este proceso los asistentes a los talleres de capacitación pudieron identificar las características propias desde la individualidad a lo colectivo construyendo posturas de identidad a partir de estas definiciones, posteriormente dentro del trabajo se realizó la identificación de características propias de cada uno de los grupos sociales en el que se movilizan, permitiendo generar dinámicas de autoreconocimiento como individuos y miembros de un grupos social, que pertenece a un municipio con interés de mantener la transferencia generacional con impacto en el campo.

Se abordaron características propias de su contexto que fueron identificadas por cada uno de los participantes, esto con el objetivo de apropiar y liderar procesos de intercambio y visibilidad para valorar el campo y sus rasgos culturales que permite generar procesos de sentido de pertenencia con el territorio.

Datos de población: los talleres fueron dirigidos a los asociados y a jóvenes rurales, miembros de diferentes asociaciones productoras del Departamento, en su gran mayoría provenientes de colegios agropecuarios, los cuales se caracterizan por ser líderes de la zona de impacto, con interés en mantener presente su cotidianidad en el agro y de posicionar sus productos que cosechan y transforman, de forma muy empírica. Es por ello, que reclaman ese acompañamiento de transformación láctea y cárnica que se vienen ofertando en las plantas piloto de la Universidad, con apoyo de la Vicerrectoría de Investigaciones.

Logros: Por medio de los talleres se logró capacitar a los asistentes frente a la necesidad de permanecer en los procesos agropecuarios y culturales de la región. Con una visión universal del campo y sus intereses a futuro, con una idea clara del posicionamiento de marca e identidad propia.

Inconvenientes: Los talleres no contaron con ningún tipo de contratiempos para su buen desarrollo y cumplimiento del cronograma de actividades trazado. Es importante resaltar que son muchas las asociaciones productoras que demuestran interés en seguir participando de este tipo de procesos, en la 
cual la Universidad del Quindío, se acerca a los productores. La idea es vincular un mayor número de personas y trabajar de forma armónica con otros municipios.

Perspectivas para futuros proyectos: los participantes se mostraron interesados en mantener un proceso activo en dos perspectivas: una, los procesos de transformación agroindustrial, refiriéndose permanente a experiencias en las plantas piloto tanto de la UQ, como de las instalaciones en los colegios y veredas.

Segundo, los participantes se mostraron interesados en mantener una participación activa en los procesos comunicaciones a partir de diferentes herramientas como lo son las comunidades virtuales, nuevas tecnologías, además de generar un proceso de narrativa digital y sus experiencias de tradición oral. 


\section{REFERENCIAS}

Aruguete, G. (2001). Gestión en Organizaciones del Tercer Sector. EN: Jornadas en la Universidad Di Tella de Buenos Aires, Argentina.

Boissier, S (2010). Decodificando el desarrollo del siglo XXI: Subjetividad, Complejidad, Sinapsis, Sinergía, Recursividad, Liderazgo y anclaje Territorial. San Sebastián Cantabria España.

Boisier S, (2002), Actores, Asociatividad y Desarrollo Territorial en la sociedad del conocimiento. Acerca de la propuesta de "clusterización” del Estado de Minas Gerais, Brasil.

Casado \& Gómez F.G. (2010). "Liderazgo Asociativo o cómo mandar obedeciendo”. Cuaderno práctico número 6. Segunda edición.

Martelli, A and Cachiba, S. (2020). Mitigating measures carried out by the environment secretariat of the Municipality of Itapira-SPin relation to greenhouse gases. South Florida Journal of Development, Miami, v.1,n.1,11-20, jan./mar.

Pérez, J. F. M. (1999). Factores que dificultan el aprendizaje organizativo. InLa gestión de la diversidad: XIII Congreso Nacional, IX Congreso Hispano-Francés, Logroño (La Rioja), 16, 17 y 18 de junio, 1999 (pp. 671-676). Universidad de La Rioja

Rodríguez, R (2000). El palimpsesto de la ciudad. Una perspectiva para el abordaje del debate. La construcción colectiva de un hábitat sostenible.

Sánchez, Vázquez, J.F. (2010) "Liderazgo: Teorías y aplicaciones". Salamanca: Publicaciones de la Universidad Pontificia de Salamanca. Demiurgo Colecciones

Salanova, M., Martínez, I.M. y Llorens, S. (2005). Psicología Organizacional Positiva. En F. Palací (Coord.), Psicología de la Organización. Madrid: Pearson Prentice Hall, pp. 349-376. 